\title{
Approximate Near-Field Blast Theory: A Generalized Approach
}

by

G. J. Hutchens

Westinghouse Savannah River Company

Savannah River Site

Aiken, South Carolina 29808

This paper was prepared in connection with work done under the above contract number with the U. S. Department of Energy. By acceptance of this paper, the publisher and/or recipient acknowledges the U.S. Government's right to retain a nonexclusive, royalty-free license in and to any copyright covering this paper, along with the right to reproduce and to authorize others to reproduce all or part of the copyrighted paper. 


\section{DISCLAIMER}

This report was prepared as an account of work sponsored by an agency of the United States Government. Neither the United States Government nor any agency thereof, nor any of their employees, make any warranty, express or implied, or assumes any legal liability or responsibility for the accuracy, completeness, or usefulness of any information, apparatus, product, or process disclosed, or represents that its use would not infringe privately owned rights. Reference herein to any specific commercial product, process, or service by trade name, trademark, manufacturer, or otherwise does not necessarily constitute or imply its endorsement, recommendation, or favoring by the United States Government or any agency thereof. The views and opinions of authors expressed herein do not necessarily state or reflect those of the United States Government or any agency thereof. 


\section{DISCLAIMER}

Portions of this document may be illegible in electronic image products. Images are produced from the best available original document. 


\title{
WSRC-TR-99.00320
}

Approximate near-field blast theory: a generalized approach

Gregory J. Hutchens

Westinghouse Savannah River Company

P. O. Box 616

Bldg. 703-H

Aiken, SC 29802

To be submitted to the Journal of Applied Physics

\begin{abstract}
$\underline{\text { Abstract }}$
A method for analyzing strong shock waves in arbitrary one-dimensional geometry is presented. An approximation to classical Taylor-Sedov theory is extended to the nearfield case where source mass is not negligible, accounting for differences in the chemical properties of the source mass and ambient medium. Results from example calculations are compared with previously published analytical formulae.
\end{abstract}

PACS numbers $47.40 .-\mathrm{x}, 47.40 . \mathrm{Nm}$ 


\section{INTRODUCTION}

The classical approach to analyzing the propagation of strong shocks developed by Taylor and Sedov assumes that the initial energy release, which drives the propagation, is released in an infinitesimal volume, i.e., a point source release. This assumption is valid only when the mass swept up by the shock wave, $\mathrm{m}_{0}$, is much greater than the original source mass, $\mathrm{m}_{\mathrm{s}}$, that is, $\mathrm{m}_{0} \gg \mathrm{m}_{\mathrm{s}}$. In this case it is a reasonable approximation to neglect the source mass and consider the energy release from a point.

On the other hand, applications can arise where the assumption of $m_{0} \gg m_{s}$ is not valid. Previously published analyses ${ }^{1,2,3}$ have addressed shock propagation in the nearfield where the source mass is not negligible, assuming that the chemical properties of the source mass and ambient medium are characterized by the same value for the ratio of specific heats, $\gamma$ (assumed constant). The objective here is to generalize the previously published analyses into a single, unified methodology and account for chemical differences between the source mass and ambient medium by allowing different values for $\gamma$ in the two regions.

First, an approximation to the classical Taylor-Sedov approach, ${ }^{4}$ valid when $\mathrm{m}_{0}>>\mathrm{m}_{\mathrm{s}}$, will be reviewed. Next, this approximation will be extended to the near-field region where $m_{0} \leq m_{s}$ by explicitly including the source mass. Finally, several example calculations will be performed so that the results of the generalized methodology can be compared to previously published analytical formulae to ensure the correct behavior is obtained and demonstrate potential applications. 


\section{REVIEW OF APPROXIMATE TAYLOR-SEDOV BLAST THEORY}

Analyzing a blast wave using Taylor-Sedov theory it is first assumed that the initial energy release that drives the blast wave occurs at a point, i.e., in an infinitesimal volume. Next, dimensional analysis is used to construct similarity variables that are used to reduce the Euler equations from partial differential equations to ordinary differential equations. Finally, this reduced set of differential equations is solved along with the integral expressing conservation of energy (also.written in terms of the similarity variables) for the flow behind the shock front.

To develop an approximate expression for the position of a strong shock, $R(t)$, in an arbitrary one-dimensional flow, ${ }^{4}$ begin with the integral expressing conservation of energy,

$$
\mathrm{E}_{0}=\mathrm{C}_{\mathrm{N}} \int_{0}^{\mathrm{R}(\mathrm{t})}\left(\frac{1}{2} \mathrm{u}^{2}+\mathrm{e}\right) \rho \mathrm{r}^{\mathrm{N}} \mathrm{dr}, \mathrm{N}=0,1,2
$$

where $E_{0}$ is the initial energy release, $\rho, u$, and e are the mass density, flow speed, and specific internal energy, respectively, behind the shock front, and $C_{N}$ and $N$ are geometrical factors. Specifically, for Cartesian geometry $(N=0), C_{N}=A$, where $A$ is the channel cross sectional area; for cylindrical geometry $(N=1), C_{N}=2 \pi L$, where $L$ is the charge length; and for spherical geometry $(N=2), C_{N}=4 \pi$.

Since the expanding shock wave is assumed to be "strong," this means that the initial energy release, $E_{0}$, is sufficiently large that the internal energy of the ambient medium is negligible. This is equivalent to neglecting the ambient pressure, $\mathrm{P}_{0}$. This allows the Rankine-Hugoniot relations to be written in the form for infinite shock strength 


$$
\begin{aligned}
& \rho_{1}=\frac{(\gamma+1)}{(\gamma-1)} \rho_{0}, \\
& \mathrm{u}_{1}=\frac{2}{(\gamma+1)} \dot{\mathrm{R}}, \\
& \mathrm{P}_{1}=\frac{2}{(\gamma+1)} \rho_{0} \dot{\mathrm{R}}^{2},
\end{aligned}
$$

where subscript 1 denotes quantities behind the shock front, $\rho_{0}$ is the density of the ambient medium (assumed constant), and $\dot{R}$ is the shock speed. Here it is assumed that the ambient medium is an ideal gas with constant $\gamma$,

$$
\mathbf{P}=(\gamma-1) \rho \mathbf{e} .
$$

As the shock wave propagates, the ambient medium is swept into a relatively thin mass shell immediately behind the shock front. This effect is especially pronounced for $\gamma$ close to 1 . With this in mind, the energy integral, Eq. (1), may be approximated by using values of $\rho, u$, and $P$ (after $e$ is eliminated using the equation of state, Eq. (5)) at the shock front and evaluating the integral over the thin mass shell. The thickness of the mass shell, $\Delta \mathrm{r}$, may be determined from conservation of mass, (mass contained in shell) $=($ mass swept up by shock),

$$
\mathrm{C}_{\mathrm{N}} \int_{\mathrm{R}-\Delta \mathrm{r}}^{\mathrm{R}} \rho_{1} \mathrm{r}^{\mathrm{N}} \mathrm{dr}=\mathrm{C}_{\mathrm{N}} \int_{0}^{\mathrm{R}} \rho_{0} \mathrm{r}^{\mathrm{N}} \mathrm{dr},
$$

Evaluating the integrals in Eq. (6a) and eliminating $\rho_{1}$ using Eq. (2) gives the thickness of the mass shell as

$$
\Delta r=\frac{1}{(\mathrm{~N}+1)} \frac{(\gamma-1)}{(\gamma+1)} \mathrm{R} .
$$


Since the thickness of the mass shell is small, it is assumed that $\mathrm{u}_{1}$ and $\mathrm{P}_{1}$ are constant across the mass shell and equal to their values at the shock front. With this assumption, the integral in Eq. (1) is simply the product of the energy per unit volume and the volume of the mass shell

$$
\mathrm{E}_{0}=\rho_{1}\left(\frac{1}{2} \mathrm{u}_{1}^{2}+\frac{\mathrm{P}_{1}}{(\gamma-1) \rho_{1}}\right)\left(\mathrm{C}_{\mathrm{N}} \mathrm{R}^{\mathrm{N}} \Delta \mathrm{r}\right)
$$

Now, using the Rankine-Hugoniot relations, Eqs.(2)-(4), to eliminate $\rho_{1}, u_{1}$, and $P_{1}$ and Eq. (6b) to eliminate $\Delta r$, Eq. (7) reduces to

$$
\frac{\mathrm{E}_{0}}{\rho_{0}}=\frac{\mathrm{C}_{\mathrm{N}}}{(\mathrm{N}+1)} \frac{4}{(\gamma+1)^{2}} \mathrm{R}^{\mathrm{N}+1} \dot{\mathrm{R}}^{2},
$$

which is a nonlinear ordinary differential equation that gives the shock position as a function of time, namely

$$
\mathrm{R}(\mathrm{t})=\left[\frac{(\mathrm{N}+3)(\gamma+1)}{4}\right]^{\frac{2}{(\mathrm{~N}+3)}}\left[\frac{(\mathrm{N}+1)}{\mathrm{C}_{\mathrm{N}}}\right]^{\frac{1}{(\mathrm{~N}+3)}}\left(\frac{\mathrm{E}_{0}}{\rho_{0}}\right)^{\frac{1}{(\mathrm{~N}+3)}} \frac{2}{\mathrm{t}^{(\mathrm{N}+3)}}
$$

\section{GENERALIZED APPROXIMATE NEAR-FIELD BLAST THEORY}

To derive an expression for the shock position near the source of energy release, the energy integral, Eq. (1), must be modified to include the energy of the source debris,

$$
E_{0}=C_{N} \int_{0}^{R_{c}}\left(\frac{1}{2} u_{d}^{2}+e_{d}\right) \rho_{d} r^{N} d r+C_{N} \int_{R_{c}}^{R}\left(\frac{1}{2} u_{g}^{2}+e_{g}\right) \rho_{g} r^{N} d r,
$$

where, again, $N=0,1,2$, subscript " $d$ " labels the source debris, subscript " $\mathrm{g}$ " labels the ambient medium, and $R_{c}$ is the location of the contact discontinuity between the source debris and the ambient medium swept up by the shock and is defined by 


$$
R(t)=R_{c}+\Delta r
$$

The lower limit of integration in the first integral of Eq. (10) is taken to be zero since it is assumed that the relevant dimension of the source mass, $R_{s}$, is very much smaller than any $R(t)$ of interest and is, therefore, negligible.

From the approximate analysis of Section II, the second integral of Eq. (10) is approximated by Eq. (8), namely,

$$
\mathrm{E}_{\mathrm{g}}=\frac{\mathrm{C}_{\mathrm{N}}}{(\mathrm{N}+1)} \frac{4}{\left(\gamma_{g}+1\right)^{2}} \rho_{0} \mathrm{R}^{\mathrm{N}+1} \dot{\mathrm{R}}^{2},
$$

where $\gamma_{\mathrm{g}}$ is used to identify the ratio of specific heats for the ambient medium. The first integral in Eq. (10) is approximated in the same manner as used in Section II. It is assumed that $u_{d}$ and $P_{d}$ are constant behind the contact surface and equal to their values at the contact surface. Then, using the result from shock tube theory that $\mathrm{u}$ and $\mathrm{P}$ are continuous across the contact surface, the first integral in Eq. (10) is approximated as

$$
\mathrm{E}_{\mathrm{d}}=\bar{\rho}_{\mathrm{d}}\left(\frac{1}{2} \mathrm{u}_{\mathrm{g}}^{2}+\frac{\mathrm{P}_{\mathrm{g}}}{\left(\gamma_{\mathrm{d}}-1\right) \bar{\rho}_{\mathrm{d}}}\right)\left(\frac{\mathrm{C}_{\mathrm{N}}}{(\mathrm{N}+1)} \mathrm{R}^{\mathrm{N}+1}\right)
$$

where $\bar{\rho}_{\mathrm{d}}$ is the average debris density and, for small $\Delta \mathrm{r}, \mathrm{R}_{\mathrm{c}} \approx \mathrm{R}(\mathrm{t})$.

The average debris density, $\bar{\rho}_{\mathrm{d}}$, is defined from conservation of source mass

$$
\bar{\rho}_{\mathrm{d}}=\rho_{\mathrm{s}}\left(\frac{\mathrm{R}_{\mathrm{s}}}{\mathrm{R}}\right)^{\mathrm{N}+1}
$$

where $\rho_{s}$ and $R_{s}$ are the initial source density and radius (or thickness, for the case of Cartesian geometry, $N=0$ ), respectively. Physically, Eq. (12) states that the source debris mass within some distance $R(t)$ is equal to the initial source mass. 
Substituting Eq. (12) into Eq. (11) and using the Rankine-Hugoniot relations, Eqs. (3) and (4), to eliminate $u_{g}$ and $P_{g}$ leads to

$$
\mathrm{E}_{\mathrm{d}}=\frac{\mathrm{C}_{\mathrm{N}}}{(\mathrm{N}+1)}\left[\frac{2 \rho_{\mathrm{s}} \mathrm{R}_{\mathrm{s}}^{\mathrm{N}+1}}{\left(\gamma_{\mathrm{g}}+1\right)^{2}} \dot{\mathrm{R}}^{2}+\frac{2 \rho_{0}}{\left(\gamma_{\mathrm{g}}+1\right)\left(\gamma_{\mathrm{d}}-1\right)} \mathrm{R}^{\mathrm{N}+1} \dot{\mathrm{R}}^{2}\right] .
$$

Now, combining Eq. (13) with Eq. (8) gives the approximation of the energy integral for near-field blast theory, namely,

$$
\mathrm{E}_{0}=\frac{\mathrm{C}_{\mathrm{N}}}{(\mathrm{N}+1)}\left[\frac{2 \rho_{\mathrm{S}} \mathrm{R}_{\mathrm{s}}^{\mathrm{N}+1}}{\left(\gamma_{\mathrm{g}}+1\right)^{2}} \dot{\mathrm{R}}^{2}+\frac{2 \rho_{0}\left[\left(\gamma_{\mathrm{g}}+2 \gamma_{\mathrm{d}}\right)-1\right]}{\left(\gamma_{\mathrm{g}}+1\right)^{2}\left(\gamma_{\mathrm{d}}-1\right)} \mathrm{R}^{\mathrm{N}+1} \dot{\mathrm{R}}^{2}\right] \text {. }
$$

Finally, to simplify the notation, define the constant $\mathrm{K}_{1}$ and $\mathrm{K}_{2}$ as

$$
\mathrm{K}_{1}=\frac{\mathrm{C}_{\mathrm{N}}}{(\mathrm{N}+1)} \frac{2 \rho_{\mathrm{s}} \mathrm{R}_{\mathrm{s}}^{\mathrm{N}+1}}{\left(\gamma_{\mathrm{g}}+1\right)^{2}}, \quad \mathrm{~K}_{2}=\frac{\mathrm{C}_{\mathrm{N}}}{(\mathrm{N}+1)} \frac{2 \rho_{0}\left[\left(\gamma_{\mathrm{g}}+2 \gamma_{\mathrm{d}}\right)-1\right]}{\left(\gamma_{\mathrm{g}}+1\right)^{2}\left(\gamma_{\mathrm{d}}-1\right)} .
$$

With these definitions Eq. (14) may be written in the following simple and convenient form

$$
E_{0}=\left[K_{1}+K_{2} R^{N+1}\right] \dot{R}^{2} .
$$

Just as with Eq. (8), Eq. (16) is a nonlinear first-order ordinary differential equation for the shock position, $\mathrm{R}$, as a function of time and may be written as

$$
\frac{\mathrm{dR}}{\mathrm{dt}}=\frac{\mathrm{E}_{0}^{1 / 2}}{\left[\mathrm{~K}_{1}+\mathrm{K}_{2} \mathrm{R}^{\mathrm{N}+1}\right]^{1 / 2}} .
$$

\section{NUMERICAL EXAMPLES}

A series of example calculations have been performed to demonstrate potential applications of Eq. (17). First, a single problem is solved for all three geometries and the 
numerical results compared with the previously published exact solutions. This will allow validation of the computer code used to solve Eq. (17) and examine its accuracy as well as verify that previously published results are obtained from the generalized methodology for the special case of $\gamma_{\mathrm{d}}=\gamma_{\mathrm{g}}$. Then a separate problem is solved for the case of Cartesian geometry. In this problem the effect of different $\gamma_{\mathrm{d}}$ and $\gamma_{\mathrm{g}}$ may be examined.

To establish the accuracy of the numerical solution of Eq. (19) and validate that the proper behavior is obtained, three example calculations were performed using the following data: $E_{0}=1 \times 10^{6} \mathrm{~J}, \rho_{0}=1 \times 10^{-3} \mathrm{~kg} / \mathrm{m}^{3}, \rho_{s}=200 \mathrm{~kg} / \mathrm{m}^{3}, R_{\mathrm{s}}=1 \times 10^{-3} \mathrm{~m}, \gamma_{\mathrm{d}}=\gamma_{\mathrm{g}}=$ 1.67, $\mathrm{A}=1 \mathrm{~m}^{2}, \mathrm{~L}=1 \mathrm{~m}$, and $\mathrm{N}=0,1,2$. For reference, the previously published exact solutions are: for Cartesian geometry $(\mathrm{N}=0)$

$$
\begin{aligned}
& \mathrm{R}=\frac{1}{\mathrm{C}_{2}}\left[\left(\frac{3}{2} \mathrm{C}_{2} \mathrm{E}_{0}^{1 / 2} \mathrm{t}+\mathrm{C}_{1}^{3 / 2}\right)^{2 / 3}-\mathrm{C}_{1}\right], \\
& \mathrm{C}_{1}=\frac{\mathrm{A}}{8}\left(\frac{2}{\gamma+1}\right)^{2} \rho_{\mathrm{s}} \mathrm{R}_{\mathrm{s}} \\
& \mathrm{C}_{2}=\frac{\rho_{0} \mathrm{~A}}{(\gamma+1)}\left(\frac{1}{\gamma-1}+\frac{4}{\gamma+1}\right),
\end{aligned}
$$

which is Eq. (16) of Reference 1; for cylindrical geometry $(\mathrm{N}=1)$

$$
\begin{aligned}
2 \sqrt{E_{0}} t & =\frac{C_{1}}{\sqrt{C_{2}}}\left\{\left(\frac{C_{2}}{C_{1}}\right)^{1 / 2} R \sqrt{1+\left(\frac{C_{2}}{C_{1}}\right) R^{2}}+\ln \left[\sqrt{1+\left(\frac{C_{2}}{C_{1}}\right) R^{2}}+\left(\frac{C_{2}}{C_{1}}\right)^{1 / 2} R\right]\right\} \\
C_{1} & =\frac{2 \pi L \rho_{s} R_{s}}{(\gamma+1)^{2}},
\end{aligned}
$$




$$
\mathrm{C}_{2}=\frac{2 \pi \mathrm{L} \rho_{0}(3 \gamma-1)}{\left(\gamma^{2}-1\right)(\gamma+1)}
$$

which is Eq. (21) of Reference 2; and, for spherical geometry ( $N=2$ )

$$
\begin{aligned}
& \sqrt{\mathrm{E}_{0}} \mathrm{t}=\mathrm{C}_{1}^{1 / 2}\left(\frac{\mathrm{C}_{1}}{\mathrm{C}_{2}}\right)^{1 / 2}\left(\frac{3}{5} \frac{1}{3^{1 / 4}} \mathrm{~F}\left(\phi, 75^{0}\right)+\frac{2}{5} \mathrm{x}\left(1+\mathrm{x}^{3}\right)^{1 / 3}-0.842\right), \\
& \mathrm{x}=\left(\frac{\mathrm{C}_{2}}{\mathrm{C}_{1}}\right)^{1 / 3} \mathrm{R}, \quad \phi=\cos ^{-1}\left(\frac{\sqrt{3}-1-\mathrm{x}}{\sqrt{3}+1+\mathrm{x}}\right), \\
& \mathrm{C}_{1}=\frac{8}{3} \frac{\pi}{(\gamma+1)^{2}} \rho_{\mathrm{s}} \mathrm{R}_{\mathrm{s}}^{3}, \\
& \mathrm{C}_{2}=\frac{8 \pi}{3} \rho_{0}\left(\frac{2}{(\gamma+1)^{2}}+\frac{1}{\gamma^{2}-1}\right),
\end{aligned}
$$

which is Eq. (30) of Reference 3.

The initial comparison for the case of Cartesian geometry showed very poor agreement with relative error between the exact and numerical solutions ranging between $30 \%$ to $90 \%$. A review of the derivation of Eq. (18) showed an additional approximation not used here, namely, at the contact surface $u_{c}=\frac{1}{2} u_{g}, P_{c}=\frac{1}{2} P_{g}$. This will make the constants $C_{1}$ and $C_{2}$ used in Eq. (19) different from $K_{1}$ and $K_{2}$ for Cartesian geometry (N $=0$ ). By using the following scaling on $C_{1}$ and $C_{2}$, the effect of the additional approximation may be removed,

$$
\overline{\mathrm{C}}_{1}=4 \mathrm{C}_{1}, \overline{\mathrm{C}}_{2}=\frac{2(3 \gamma-1)}{(5 \gamma-3)} \mathrm{C}_{2}
$$

Using $\overline{\mathrm{C}}_{1}$ and $\overline{\mathrm{C}}_{2}$ in Eq. (18) results in excellent agreement with the numerical solution. This is shown in Fig. 1. 
The comparison for the case of cylindrical geometry showed excellent agreement between Eq. (19) and the numerical solution. This is shown in Fig. 2.

Finally, the initial comparison for the case of spherical geometry showed a uniform relative error of greater than $450 \%$. A review of the derivation of Eq. (20) showed a typographical error in the published solution not identified in the Erratum. ${ }^{3}$ The term $\left(\frac{C_{1}}{C_{2}}\right)^{1 / 2}$ should be $\left(\frac{C_{1}}{C_{2}}\right)^{1 / 3}$. Making this correction in Eq. (20) resulted in excellent agreement with the numerical solution. This is shown in Fig. 3.

As an example application of Eq. (17), data from Reference 1 is used to examine the effect of different values of $\gamma_{\mathrm{d}}$ and $\gamma_{\mathrm{g}}$ on shock propagation. The specific data used are $E_{0}=2.28 \times 10^{6} \mathrm{~J}, \rho_{0}=1.205 \mathrm{~kg} / \mathrm{m}^{3}, \rho_{\mathrm{s}}=1.8 \times 10^{3} \mathrm{~kg} / \mathrm{m}^{3}, \mathrm{R}_{\mathrm{s}}=2.54 \times 10^{-2} \mathrm{~m}$, and $\mathrm{A}=$ $7.854 \times 10^{-3} \mathrm{~m}^{2}$. The first example assumes a constant value for $\gamma_{\mathrm{g}}$ (equal to 1.4) and varies $\gamma_{\mathrm{d}}$. These results are shown in Fig. 4. The second example assumes a constant value for $\gamma_{\mathrm{d}}$ (equal to 3 , which is typical of explosives in the density range of 1 to 1.8 $\left.\mathrm{g} / \mathrm{cm}^{3}\right)^{5,6}$ and varies $\gamma_{\mathrm{g}}$. These results are given in Fig. 5 .

The results given in Fig. 4 show that varying $\gamma_{d}$ while keeping $\gamma_{g}$ constant has a marked effect on shock propagation. In the previous analysis for Cartesian geometry, Eq. (18), only curve "b" of Fig. 4 would be obtained. For a constant $\gamma_{g}$, an increase in $\gamma_{d}$ will lead to faster shock propagation due to an increase in the driving pressure in the source debris. This can be seen from the equation of state, Eq. (5). For a given value of internal energy per unit volume ( $\rho e$ ) an increase in $\gamma_{d}$ leads to an increase in pressure since higher $\gamma_{d}$ means more internal energy is in the translational degrees of freedom of the source debris. 
The results given in Fig. 5 show that varying $\gamma_{g}$ while keeping $\gamma_{d}$ constant also affects shock propagation, as expected, but to a lesser degree. As $\gamma_{\mathrm{g}}$ increases less energy is deposited in the internal degrees of freedom. This increases the average energy of the particles in the ambient medium, which increases the rate of momentum transfer, resulting in faster shock propagation.

\section{CONCLUSION}

A generalized methodology for analyzing strong shocks in arbitrary one-dimensional geometry, accounting for differences in the chemical properties of the source debris and ambient medium, has been developed. Numerical results from the generalized method show excellent agreement with previously published analytical formulae for Cartesian, cylindrical, and spherical geometry. Now that the methodology has been derived and implemented numerically, additional physics, such as plasma effects or radiation, can be added, as required, to improve the utility of the overall methodology.

The generalized methodology will be useful in a variety of applications. For example, in Cartesian geometry it can be used to analyze data from explosive driven shock tube experiments. In cylindrical geometry it can be used to analyze data from exploding wire experiments or in the preliminary design of pressure vessels. Finally, in spherical geometry it can be used for preliminary design of inertial confinement fusion chambers. 


\section{ACKNOWLEDGEMENTS}

The author gratefully acknowledges helpful review and comments from B. J. Hardy of Westinghouse Savannah River Company and insights on incomplete elliptic integrals from S. C. King of the University of South Carolina at Aiken.

\section{REFERENCES}

${ }^{\text {I} D . ~ A . ~ F r e i w a l d, ~ " A p p r o x i m a t e ~ b l a s t ~ w a v e ~ t h e o r y ~ a n d ~ e x p e r i m e n t a l ~ s h o c k ~ t r a j e c t o r i e s ~ i n ~}$ linear explosive driven shock tubes," J. Appl. Phys., $\underline{43}$, no. 5, p. 2224, (1972).

${ }^{2}$ G. J. Hutchens, “Approximate cylindrical blast theory: Near-field solutions," J. Appl. Phys., 77, no. 7, p. 2912, (1995).

${ }^{3}$ D. A. Freiwald and R. A. Axford, "Approximate spherical blast theory including source mass," J. Appl. Phys., 46, no. 3, p. 1171, (1975). See also "Erratum: Approximate spherical blast theory including source mass, [J. Appl. Phys., 46, no. 3, p. 1171, (1975)]," J. Appl. Phys., 46, no. 8, p. $3697,(1975)$.

${ }^{4}$ E. G. Harris, "Exact and approximate treatment of the one-dimensional blast wave," Naval Research Laboratory Report 4858, (1956).

${ }^{5}$ F. H. Harlow and A. A. Amsden, "Fluid Dynamics: A LASL Monograph," LA-4700, (1971).

${ }^{6}$ P. W. Cooper, Explosives Engineering, Wiley-VCH: New York, (1996). 
FIG. 1. Percent relative error as a function of time for Cartesian geometry $(N=0)$

FIG. 2. Percent relative error as a function of time for cylindrical geometry $(\mathrm{N}=1)$

FIG. 3. Percent relative error as a function of time for spherical geometry $(\mathrm{N}=2)$

FIG. 4. Shock position as a function of time for $\gamma_{\mathrm{g}}=1.4$ : a) $\gamma_{d}=1.2$; b) $\gamma_{d}=1.4$; c) $\gamma_{d}=$ $1.67 ;$ d) $\gamma_{d}=3.0$

FIG. 5. Shock position as a function of time for $\gamma_{d}=3.0$ : a) $\gamma_{g}=1.2$; b) $\gamma_{g}=1.4$; c) $\gamma_{g}=$ 1.67 


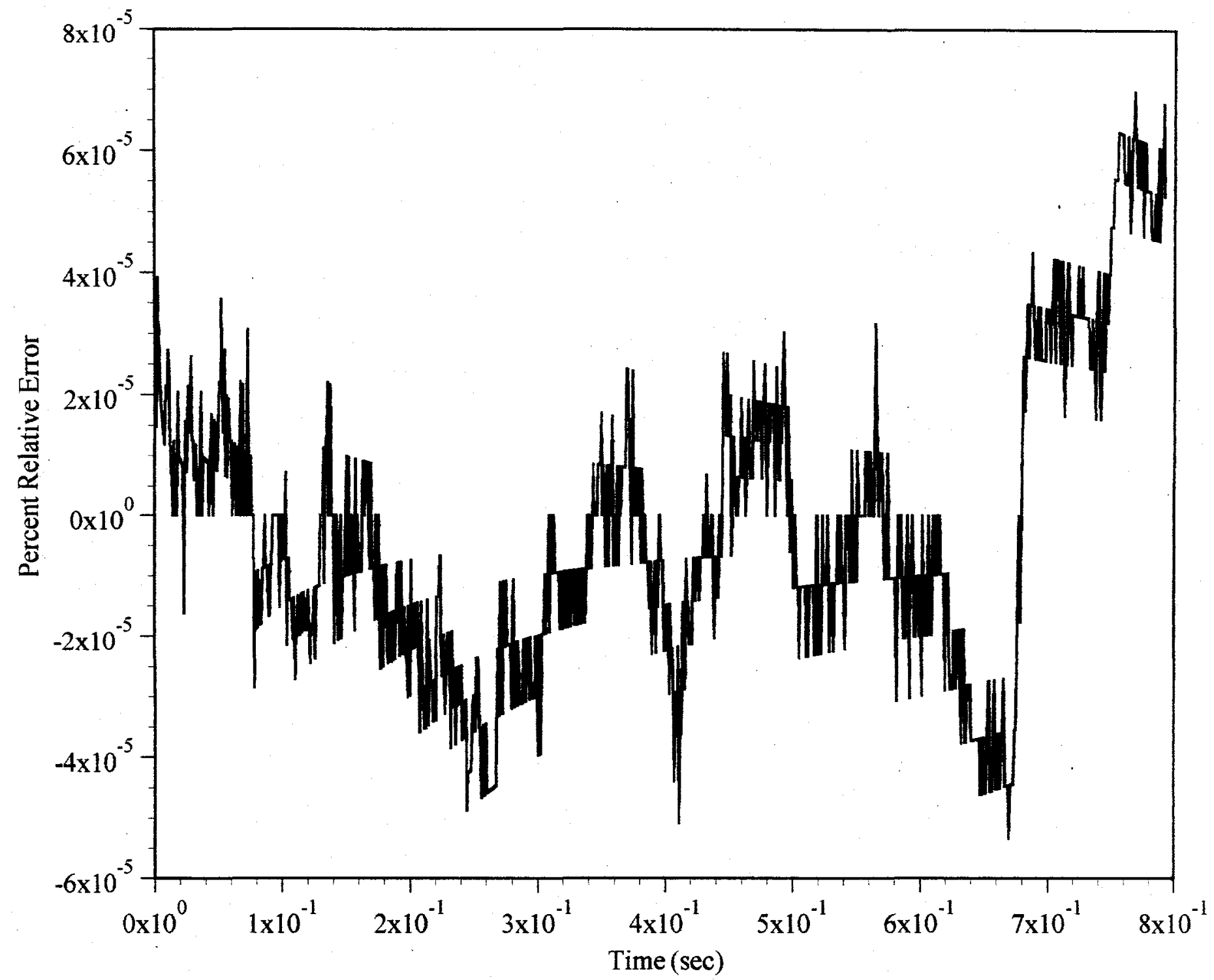

Fig.l 


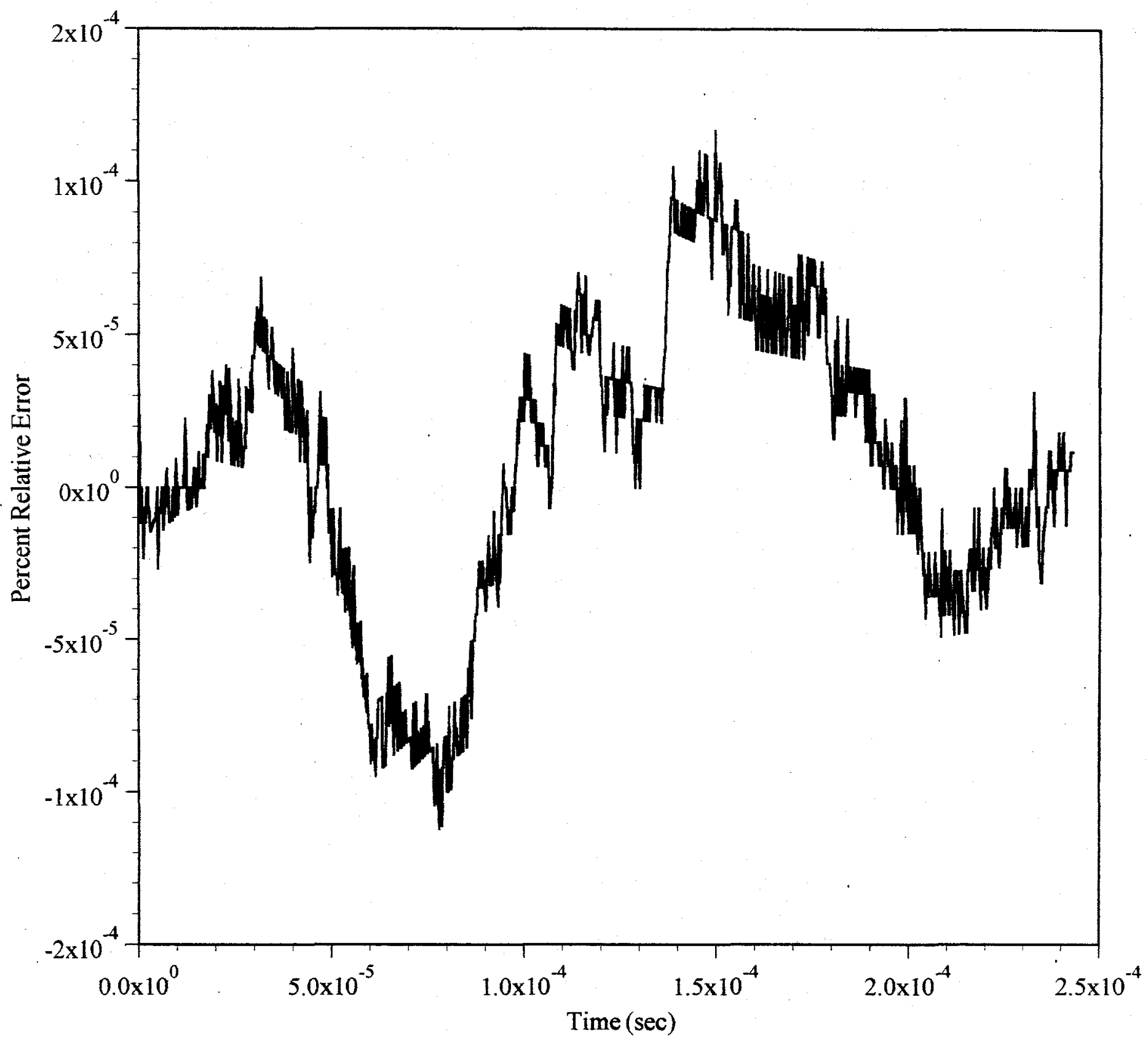

$F_{\text {ig. } 2}$ 


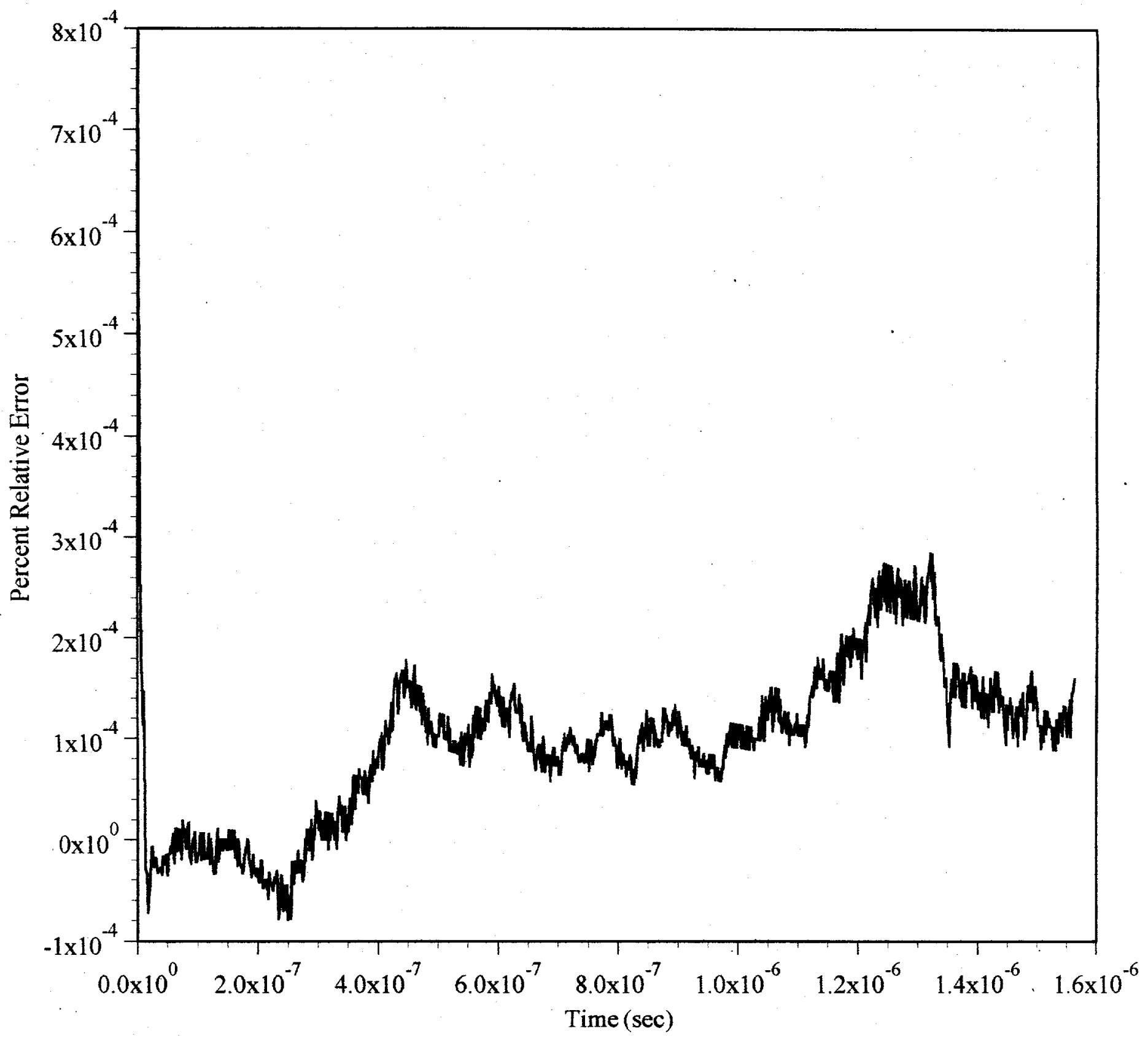




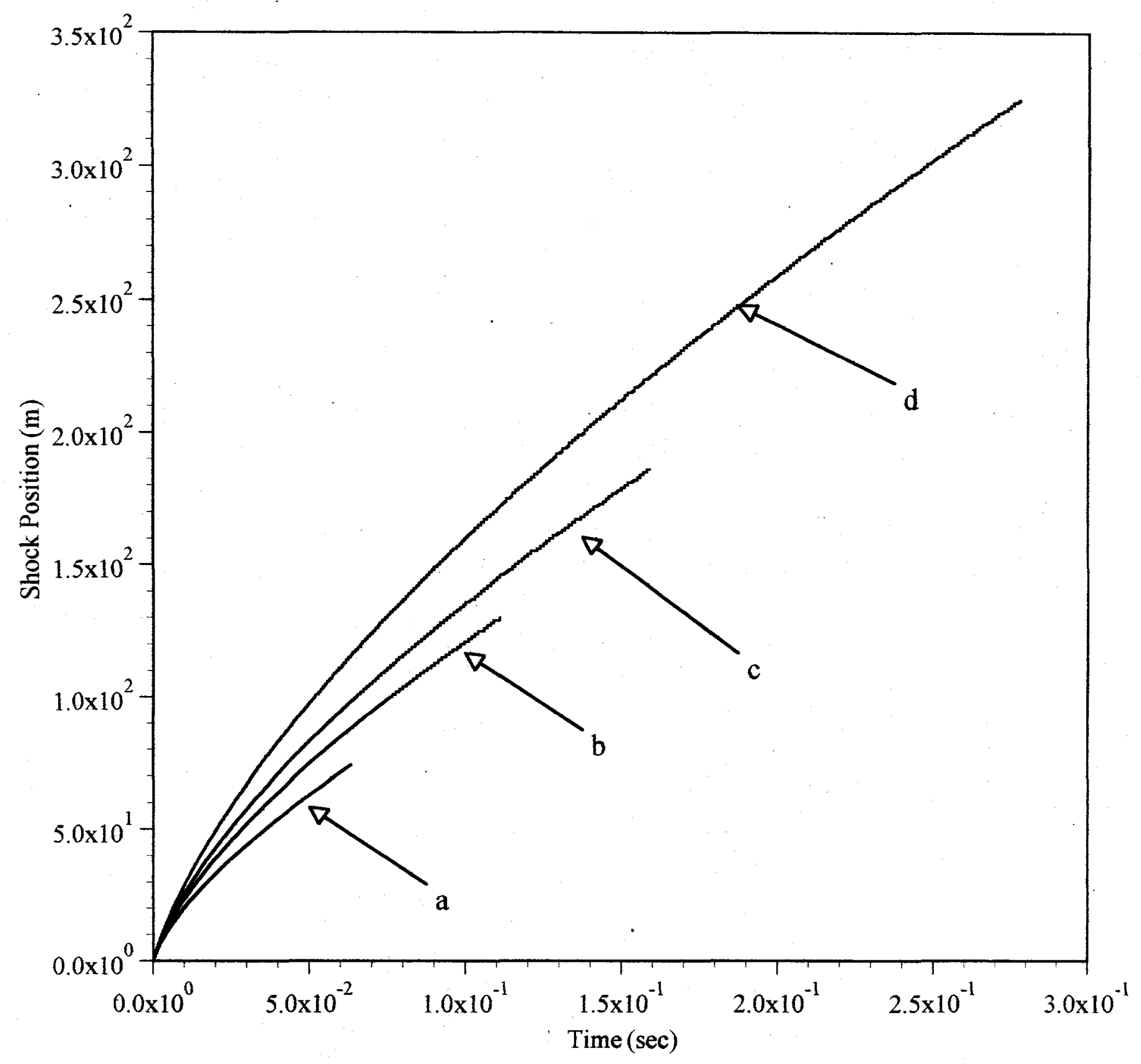

Fig. 4 


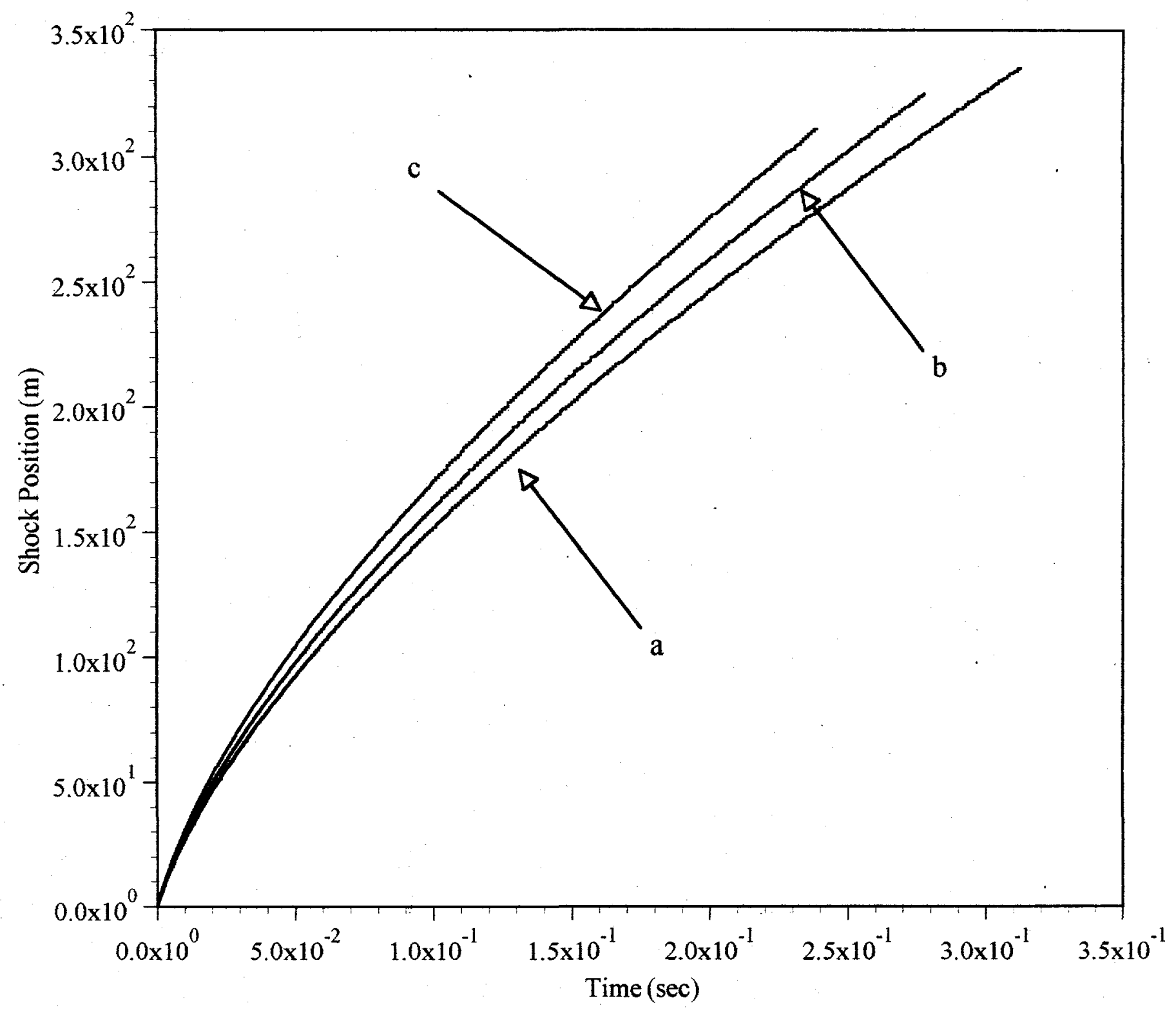

Fig.s 